\title{
Deuterium thermal desorption from carbon based materials: a comparison of plasma exposure, ion implantation, gas loading, and C-D codeposition.
}

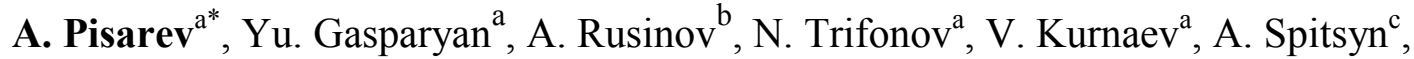 \\ B. Khripunov ${ }^{\text {, }}$ T. Schwarz-Selinger ${ }^{d}$, M. Rasinski ${ }^{\mathrm{e}}$, K. Sugiyama ${ }^{\mathrm{d}}$ \\ ${ }^{a}$ National Research Nuclear University "MEPHI”, Kashirskoe sh. 31, Moscow, 115409, Russia. \\ ${ }^{b}$ Kyushu University, Interdisciplinary Graduate School of Engineering Sciences, Kasuga Koen, 6-1, \\ Kasuga, Fukuoka, 816-8580, Japan. \\ ${ }^{c} R R C$ Kurchatov Institute, Ac. Kurchatov sq., 1/1, Moscow, 123182, Russia. \\ ${ }^{d}$ Max-Plank-Institut für Plasmaphysik, EURATOM Association, Boltzmanstr.2, D-85748, \\ Garching, Germany \\ ${ }^{e}$ Warsaw University of Technology, Faculty of Material Science and Engineering, Woloska 141, 02- \\ 507, Poland

\begin{abstract}
Thermal desorption spectra from fine grain graphite and carbon fiber composite exposed in different plasma installations have been compared with those obtained after ion implantation, absorption from gas, and those from soft and hard C-D films. Features of the spectra were analysed and led to the conclusion that ion implantation, absorption, and amorphous C:D layer contribute to trapping during plasma irradiation.
\end{abstract}

JNM Keywords: Carbon-based materials, Co-deposition, Deuterium implantation, Deuterium retention, Thermal desorption spectra.

PSI Keywords: Carbon-based materials, Carbon deposition, Co-deposition, Deuterium implantation, Deuterium retention.

PACS: 52.40.Hf, 68.43.Vx, 81.05.Uw, 82.30.Rs

Corresponding author address: National Research Nuclear University "MEPHI”, 115409, Russia, Moscow, Kashirskoe sh. 31.

Corresponding author e-mail: pisarev@plasma.mephi.ru

Presenting author: Alexander Pisarev

Presenting author e-mail: pisarev@ plasma.mephi.ru 


\section{Introduction}

Use of carbon based materials in ITER is questioned mainly due to possible high tritium inventory. An overview on hydrogen trapping and release is given in [1]. TDS after ion implantation [2-10], plasma irradiation [11-14], loading from gas [15-17], and co deposition of C-H films are often investigated. Experimental TDS sometimes seriously differ.

This work is focused on comparison of thermal desorption spectra after irradiation in different plasmas, implantation from ion beam, loading from gas, and co-deposition of soft and hard C-D films made in one campaign.

\section{Experimental details}

Samples $10 \times 10 \times 1 \mathrm{~mm}^{3}$ were cut from fine grain graphite MPG-8 (NII GRAPHIT, Russia, $1.85 \mathrm{~g} / \mathrm{cm}^{3}$ ) and carbon fiber composite NB31 (Snecma Propulsion Solide, France, $\left.1.9 \mathrm{~g} / \mathrm{cm}^{3}\right)$. They were polished mechanically and degassed in vacuum $2 \times 10^{-5} \mathrm{mbar}$ at $1200{ }^{\circ} \mathrm{C}$ for $15 \mathrm{~min}$.

Five plasma installations were used: radio frequency discharge (RFD) [18], beam plasma discharges LENTA [19] and PR-2 [20], magnetron discharge MD [11] and ECR discharge PlaQ [21]. Ion energies were $100-200 \mathrm{eV}$, sample temperatures $50-150^{\circ} \mathrm{C}$, ion fluxes $2-30 \times 10^{20} \mathrm{D} / \mathrm{m}^{2} \mathrm{~s}$, and deuterium pressures $10^{-4}-10^{-3} \mathrm{mbar}$.

Two ion beam installations were used: MEDION (MEPHI, Moscow) with the ion energy $E=5 \mathrm{keV}$ and the flux $i=1 \times 10^{18} \mathrm{D} / \mathrm{m}^{2} \mathrm{~s}$ and HSQ (IPP, Garching) with $E=200 \mathrm{eV}$ and $\left.i=1 \times 10^{19} \mathrm{D} / \mathrm{m}^{2} \mathrm{~s}\right)$. The samples were at about room temperature.

Gas absorption was performed at $10 \mathrm{kPa}$ and temperatures $T_{\mathrm{abs}}=630$ and $830 \mathrm{~K}$ for 1 hour.

Two types of amorphous C-D films "soft" and "hard" were deposited on W substrates in $\mathrm{CD}_{4}$ plasma in PlaQ with the potential on the sample of $10-15 \mathrm{~V}$ and $100 \mathrm{~V}$, respectively.

\section{Experimental results}

About 60 samples were irradiated in different plasmas, and spectra of all D-containing molecules were compared. Spectra from MPG-8 and CFC are similar in shape but different in D content [22]. All spectra after irradiation by different plasmas, Figure 1, are qualitatively 
similar. They are in the range of $400-1500 \mathrm{~K}$ with maximum around $800 \mathrm{~K}$ at high fluence, which decreased at low fluence [22].

One can distinguish four broad "maxima" 450-550, 750-850, 950-1000, and 1200$1300 \mathrm{~K}$. Their shapes are variable, which is possible if the maxima consist of several nonresolved peaks. Narrower peaks are often better seen for masses 3, 19, and 20 (HD, $\left.\mathrm{HDO}+\mathrm{CHD}_{3}, \mathrm{D}_{2} \mathrm{O}+\mathrm{CD}_{4}\right)$. Accurate deconvolution of the spectra in separate peaks is almost impossible as it needs a knowledge about mechanisms of desorption, which determine peak shapes.

Though, basic parameters of irradiation in different plasmas were similar, the relative amplitudes of peaks are different. RF discharge gives a rather narrow peak $800 \mathrm{~K}$, which always dominates. Irradiation in magnetron discharge gives additionally intensive high temperature peaks and pronounced low temperature peaks of $\mathrm{D}_{2}$ and rather high $\mathrm{CD}_{4}$ release, contrary to RF, LENTA, and PR-2. High temperature peak is also large after irradiation in PlaQ.

There are many factors that complicate the experiments with plasma. Among them are: correct D flux measurements, ion and electron stimulated processes, development of the relief, impurities, and film deposition. Another factor is non uniformity of carbon based materials that was well demonstrated in [23] in gas driven permeation experiments with graphite wafers cut from different parts of the same graphite block. Therefore, it is not surprising that results after irradiation in different facilities differ though general features of the spectra are about the same. Sometimes, even samples irradiated simultaneously gave different spectra.

Spectra after ion implantation, Figure 2, lay in a range 700-1600 K and 600-1500 K for $5 \mathrm{keV}$ and $200 \mathrm{eV}$ deuterons, respectively. Even at $200 \mathrm{eV}$, spectra are very different from those obtained after plasma irradiation. There is no prominent peak at $800 \mathrm{~K}$, typical even for low fluence in plasma experiments; desorption is observed mainly at high temperatures 1000$1400 \mathrm{~K}$, where it is commonly small in experiments with plasma; release of hydrocarbons, typical for plasma irradiation, is rather small. TDS are always very smooth. Nevertheless, comparison of $\mathrm{HD}$ and $\mathrm{D}_{2}$ spectra in different conditions leads to the suggestion that at least three "peaks" around $950 \mathrm{~K}, 1150 \mathrm{~K}$, and 1350K exist.

There were many early experiments with ions described in literature. TDS of $\mathrm{D}_{2}$ were usually very broad from $700 \mathrm{~K}$ to $1300-1500 \mathrm{~K}[5,6,10]$. A wide peak around $1000-1100 \mathrm{~K}$ was often observed $[4,6]$ as in our experiments. Sometimes the temperature of the peak was different (950K [3], $1200 \mathrm{~K}$ [7]). TDS often had faint peculiarities and were deconvoluted 
into two peaks $[4,10]$. Two peaks [8] and three peaks $(1000,1100$, and $1200 \mathrm{~K})[6]$ in TDS were also observed.

TDS of deuteromethane in experiments with ions has one narrow peak at $870 \div 920 \mathrm{~K}$, which is similar to the range of $850-950 \mathrm{~K}$ described in $[3,5,6,10]$. Methane spectra after plasma irradiation are much broader, consist of 2-3 peaks, and the main peak position is about $100 \mathrm{~K}$ less than in the case of ion implantation.

Comparison of spectra after ion implantation and after plasma irradiation gives a strong feeling that ion implantation experiments do not reproduce the features of experiments with plasma even in the case the ion energies are the same $(200 \mathrm{eV})$. This is possible if not only plasma ions but also hot atoms and gas molecules contribute to trapping.

The temperature range of TDS after absorption from gas is as broad as after plasma irradiation. Examples of $\mathrm{D}_{2}$ TDS after absorption at $10 \mathrm{kPa}$ for 1 hour at $T_{\mathrm{abs}}=630 \mathrm{~K}$ and $830 \mathrm{~K}$ are shown in Figure 3. Desorption of HD is higher than $\mathrm{D}_{2}$ in this case. There are well separated maxima at $600,800,1000$, and $1300 \mathrm{~K}$ in Fig. 3. Desorption after $1400 \mathrm{~K}$ is also observed. Also a weak peak at $500 \mathrm{~K}$ can be suggested though it is very small as the temperature of sample loading was higher than $500 \mathrm{~K}$. At $T_{\mathrm{abs}}=630 \mathrm{~K}$, desorption peaks around $800 \mathrm{~K}$ as in most plasma experiments; at $T_{\mathrm{abs}}=830 \mathrm{~K}$, deuterium releases at higher temperatures, which is typical for experiments with ions.

Peaks at about the same temperatures and similar shift of TDS with increase of $T_{\mathrm{abs}}$ were observed in [15]: there were only peaks with $T_{\mathrm{m}}=550 \mathrm{~K}$ and $750 \mathrm{~K}$ at $T_{\mathrm{abs}}=473 \mathrm{~K}$, but peaks with $T_{\mathrm{m}}=950 \mathrm{~K}$ and $1200 \mathrm{~K}$ appeared at $T_{\mathrm{abs}}=650 \mathrm{~K}$ and $1100 \mathrm{~K}$, respectively. Loading at higher temperatures of $1500 \mathrm{~K}$ [16] and $1170 \mathrm{~K}$ [17] led to release at even higher temperatures in the interval of $1000-1500 \mathrm{~K}$ and $1100-2100 \mathrm{~K}$ respectively.

Examples of TDS from amorphous "soft" and "hard" C-D films are given in Figure 4. Spectra of both $\mathrm{D}_{2}$ and $\mathrm{CD}_{4}$ from the two films are similar to those observed in [24]. The two films demonstrate completely different spectra. TDS from soft films are very narrow: all D containing species $\left(\mathrm{D}_{2}, \mathrm{HD}, \mathrm{C}_{\mathrm{x}} \mathrm{D}_{\mathrm{y}}\right.$, and HDO) are peaked at $750 \mathrm{~K}$. The release is due to decomposition of the film, which completely disappeared after TDS measurements. TDS of $\mathrm{D}_{2}$ from hard films have the main peak at $850 \mathrm{~K}$, and a wide high temperature part with several peaks as in experiments with plasmas. All deuterocarbons $C_{x} D_{y}(x, y=1,2,3)$ release in a single broad peak $T_{\mathrm{m}}=850 \mathrm{~K}$.

SEM analyses of plasma irradiated surfaces demonstrate that they are modified, Figure 5. The modified layer looks like a film, which is possibly important in deuterium trapping and release. 


\section{Discussions}

All the traps in experiments with gas are natural traps. TDS peaks are in the wide range of temperatures, so that the natural trapping sites cover a whole spectrum of binding energies of radiation induced traps observed in experiments with plasmas and ions.

Variation in the TDS with the temperature of absorption was interpreted in terms of different accessibility of traps at different temperatures [15]: low energy traps are easily accessible even at low temperatures, while high energy traps are accessible only at high temperatures. However, this effect can be explained also suggesting that all traps are equally accessible all through the material. Indeed, two factors determine population of different traps: the concentrations of traps, and the temperature. Population of traps at low temperatures depends mainly on their concentrations; therefore one can conclude from experiments at $T_{\mathrm{abs}}=630 \mathrm{~K}$ that the concentration of natural traps, which give the peak at $800 \mathrm{~K}$, is higher than the concentration of traps with higher binding energies. The deuterium content in the high energy traps increases with the temperature, though depopulation becomes more intensive. This is only possible due to transport of deuterium in the bulk of grains and crystallites with subsequent trapping there.

Natural trapping sites are dangling bonds. Variety of peaks in TDS can be connected with variety of these bonds on the surface and in the bulk. There are also pores, which can accumulate deuterium both on their internal surface and in their volume.

Ion irradiation, in principle, can produce radiation induced traps with various binding energies. It is impossible to distinct between natural and radiation induced traps as all the traps are dangling bonds. Mainly high temperature TDS peaks are observed after ion implantation in our experiments and in experiments described in literature. This could lead to the conclusion that radiation defects are predominantly of high binding energies. Another explanation could be that defects with higher binding energies are preferentially populated, though the concentration of all radiation induced defects is high.

TDS after plasma irradiation are as broad as after saturation from gas, and positions of peaks correlate. TDS after plasma implantation usually have also a broad high temperature part, which dominates in experiments with ions. One can conclude that trapping during plasma implantation has features of experiments with gas and experiments with ions. Both natural and radiation induced defects participate in trapping. 
With increase of the fluence and temperature, deuterium can be trapped rather deep in graphite, as recent experiments with plasma demonstrate [25]. This can complicate analyses of TDS at high fluence.

A feature of experiments with plasma is formation of a modified/deposited layer, which looks like a film. TDS acquired after plasma irradiation are often similar to TDS from hard C-D films. One can suggest that the modified layer is similar in some way to hard C-H films, and it gives an important contribution to trapping. Hard films are usually obtained if a bias of 100-200 V is applied to the substrate for decomposition of hydrocarbons. Deuterium ions during irradiation from plasma are of the same energy that can give similar radiation effect in surface modification. Nuclear reaction deuterium depth profiling often gives a peak near the surface that may be linked with the modified layer.

\section{Summary}

Comparison of thermal desorption spectra from fine grain graphite and carbon fiber composite after plasma irradiation with those after ion implantation and gas adsorption, as well as with TDS from soft and hard C-D films has been performed in one experimental campaign. All TDS consist of several broad peaks composed of more narrow non-resolved peaks. Peak positions often correlate.

Irradiation in different plasma installations gives broad spectra from 400 to $1500 \mathrm{~K}$ with similar peak positions but different amplitudes; peak at 750-850 $\mathrm{K}$ and the high temperature part $(900-1500 \mathrm{~K})$ are common. Ion implantation, when compared to plasma, gives very different TDS even at similar ion energies and fluences: there is no intensive $800 \mathrm{~K}$ peak, and high temperature range 900-1500 K always prevails. "Soft" films completely decompose and disappear in a narrow range around $750 \mathrm{~K}$, while "hard" films give a broad spectra with intensive peak at $850 \mathrm{~K}$ and a high temperature part, which are two features of TDS after plasma irradiation. Absorption from gas gives a series of peaks at similar positions as after plasma irradiation; and increase of the temperature leads to increased population of traps with high energy.

Trapping during plasma irradiation was shown to have features of ion implantation, absorption from gas, and trapping in amorphous C:D layer. Trapping of atomic hydrogen must be added here. Natural trapping sites, which were observed in experiments with gas, cover a whole spectrum of binding energies observed in experiments with plasmas, ions, as well as with films. Radiation induced traps also have very different energies, but trapping in 
traps with higher binding energies dominates. Increase of the temperature in experiments with gas and plasma provokes deuterium trapping deeper in the material bulk.

\section{Acknowledgements}

The work was supported by the Russian Ministry for Education and Science.

\section{References}

[1] C.H.Skinner, A.A.Haasz, V.Kh.Alimov, N.Bekris, R.A.Causey, R.E.Clark, J.P.Coad, J.W.Davis, R.P.Doerner, M.Mayer, A.Pisarev, J.Roth, T.Tanabe, Fusion Science and Technology, 2008, v.54, p.891-945

[2] A.A. Haasz, J.W. Davis, J. Nucl. Mater. 209 (1994) 155

[3] M. Mayer, M.Balden, R.Behrisch, J. Nucl. Mater. 252 (1998) 55

[4] T.Hino, F.Hirano, Y.Yamauchi, Y.Hirohata, Fus. Eng. Design 49-50 (2000) 213

[5] A.A. Haasz, J.W. Davis, J. Nucl. Mater. 232 (1996) 219.

[6] H.Atsumi, S.Tokura, S.Yamanaka, et al., J. Nucl. Mater., 141-143 (1986) 113

[7] D.B.Kuzminov, S.L.Kanashenko, A.E.Gorodetsky A.P.Zakharov, J.Nucl. Mater. 185 (1991) 123

[8] C.Garcia-Rosales, J.Roth, R.Behrisch, J. Nuc. Mater. 212-215 (1994) 1211

[9] M.Oyaidzu, H.Kimura, T.Nakahata, et al., J. Nucl. Mater. 367-370 (2007) 1522

[10] Y.Morimoto, K.Okuno, J.Nucl. Mater. 313-316 (2003) 595

[11] V.Kh.Alimov, J.Roth, Phys. Scripta, T128 (2007) 6

[12] H.Yoshida, M.Taniguchi, K.Yokoyama, et al., J.Nucl.Mater., 329-333 (2004) 790

[13] M. Balden et al., Phys. Scripta T102 (2003) 38

[14] I.I. Arkhipov, A.E. Gorodetsky, R.Kh. Zalavutdinov et al., J. of Nucl. Mater. 271\&272 (1999) 418

[15] H.Atsumi, S.Tokura, M.Miyake, J. Nucl. Mater., 155-157 (1988) 241

[16] A.E.Gorodetsky, A.V.Markin, V.N.Chernikov, et al., Fus. Eng. Des. 43 (1998) 129.

[17] E.Hoinkis, J. Nucl. Mater. 183 (1991) 9

[18] A.A.Skovoroda, V.S.Svishchova, A.V.Spitsyn, et al., J. Nucl. Mater. 306 (2002) 232

[19] B. I. Khripunov, V. B. Petrov, M. I. Guseva et al., $21^{\text {st }}$ IAEA Fusion Energy Conference, Chengdu, China, 2006. Rep. EX/P4-3, Book of abstracts p.52

[20] I.V.Vizgalov, A.A.Pisarev, K.M.Gutorov, J. Nucl. Mater. 363-365 (2007) 833 
[21] B. Landkammer, A. von Keudell, W. Jacob, Journal of Nucl. Mater. 264 (1999) 48-55.

[22] A. Pisarev, T. Tanabe, B. Emmoth, et al, J. Nucl. Mater. 390-391 (2009) 677

[23] A.Spitsyn, A.Pisarev, V.Gureev, et al., J.Nucl. Mater. 363-365 (2007) 966.

[24] E.Salancon, T.Durbeck, T.Schwarz-Selinger, et al, J. Nucl. Mater. 376 (2008) 160

[25] A.Rusinov, N.Trifonov, Yu.Gasparyan, et al, Deuterium retention in graphite exposed to high flux plasma at high temperatures, J. Nucl. Mater., In press 


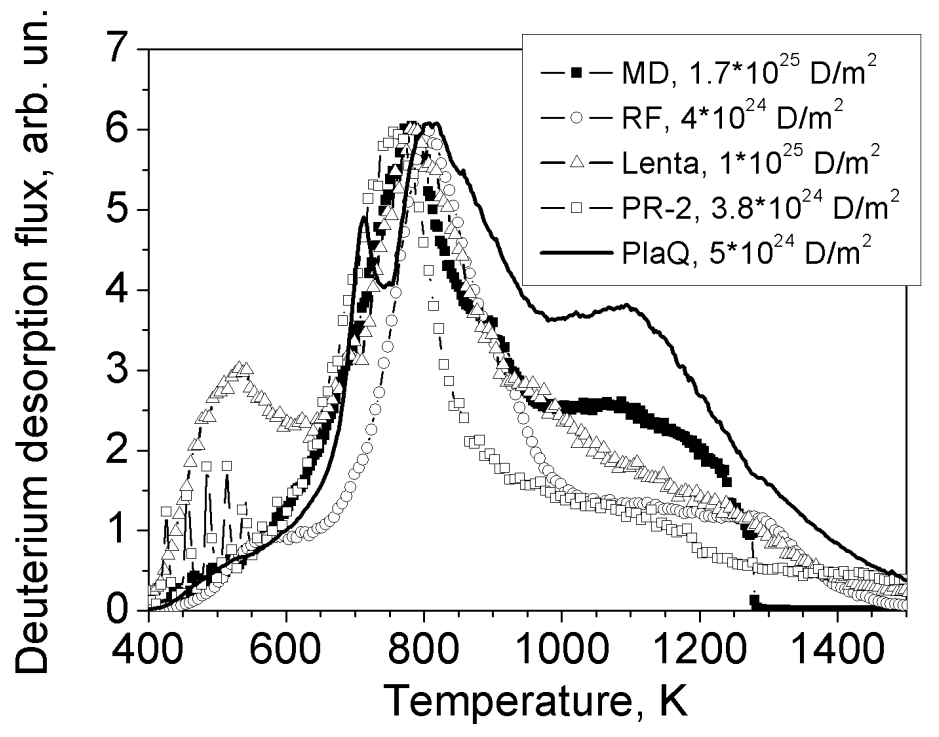

Fig.1. Comparison of TDS spectra of $\mathrm{D}_{2}$ after plasma exposure in different installations. Samples were made of MPG-8 (MD, RF, Lenta, PR-2) and CFC (PlaQ). All spectra are normalized to have the same amplitude of a maximum peak

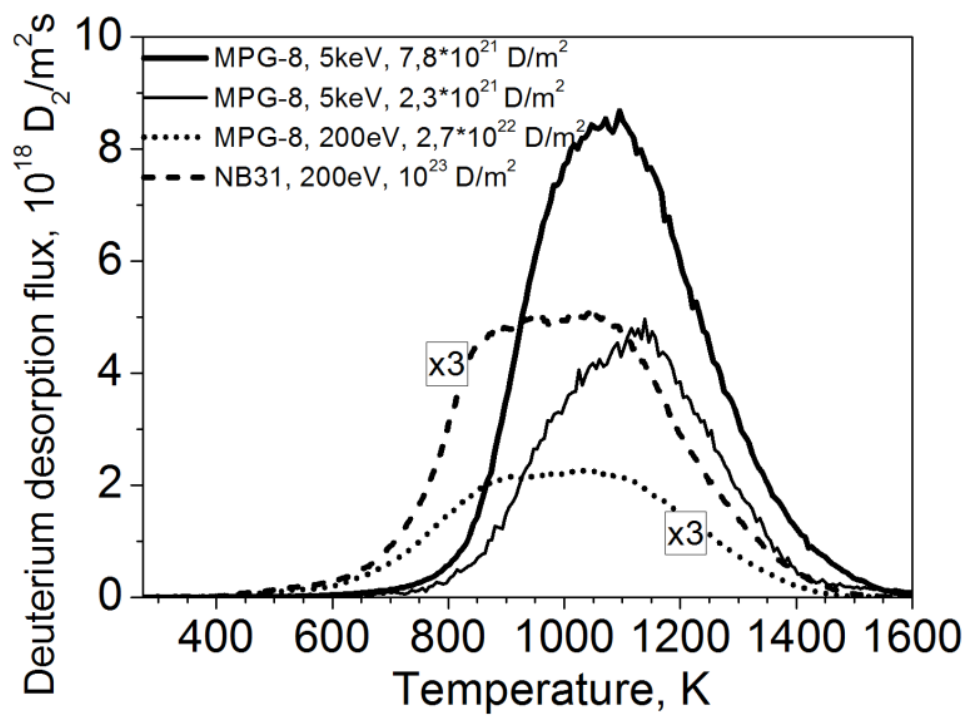

Fig. 2. TDS of $\mathrm{D}_{2}$ after ion beam bombardment. Ions with the energy of $200 \mathrm{eV} / \mathrm{D}$ ions were used in HSQ facility, while $5 \mathrm{keV} / \mathrm{D}$ ions were used in MEDION facility. Materials and fluences are given in the legend. 


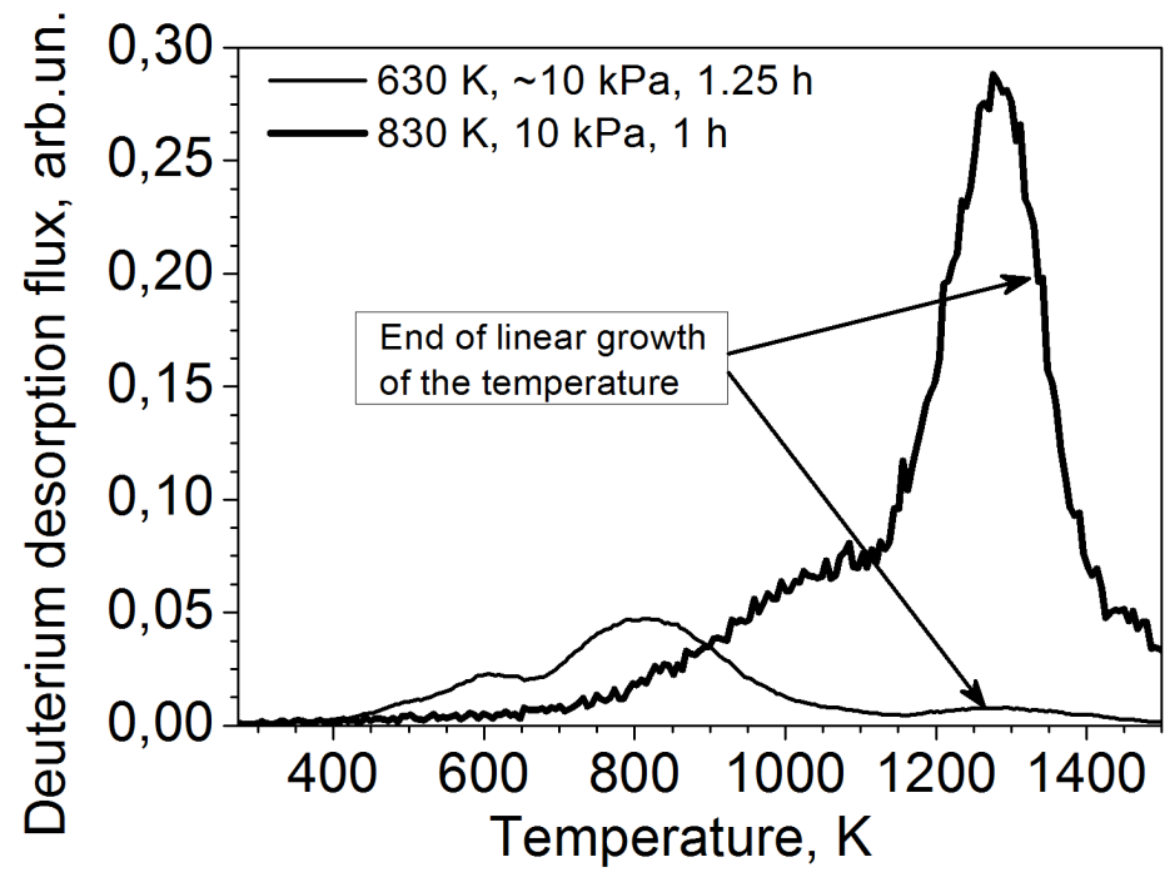

Fig. 3. TDS of $\mathrm{D}_{2}$ from MPG-8, exposed in deuterium gas at $10 \mathrm{kPa}$ at $630 \mathrm{~K}$ and $830 \mathrm{~K}$.

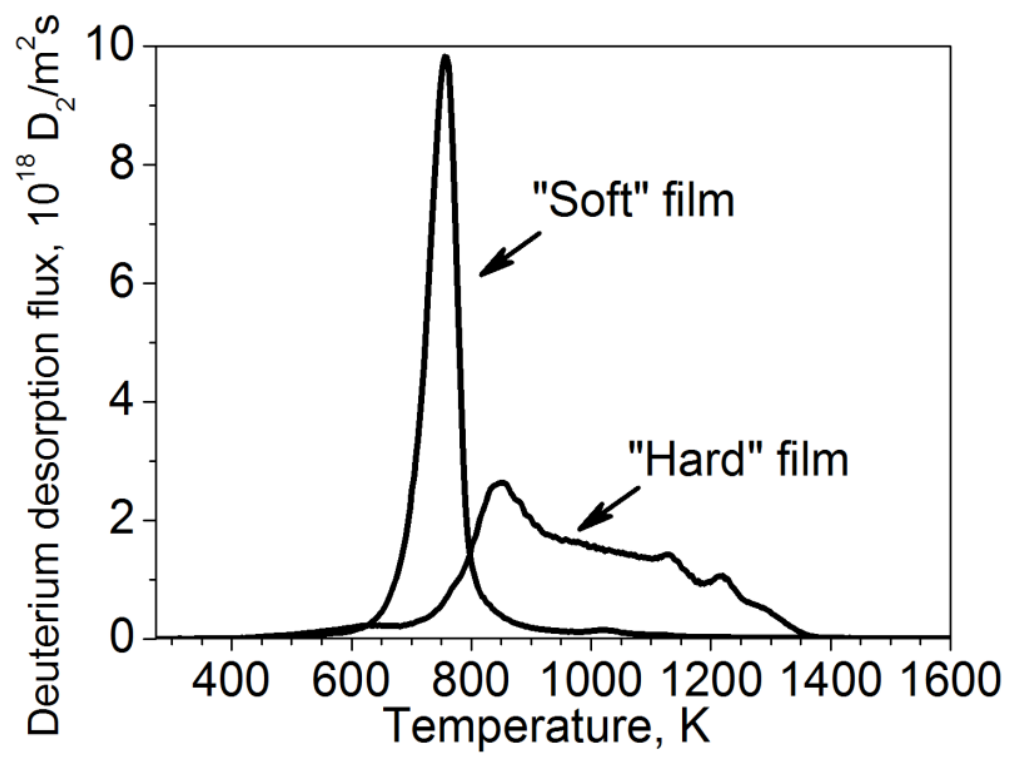

Fig.4. TDS spectra of $\mathrm{D}_{2}$ from the W sample with "soft" and "hard" C-D films 

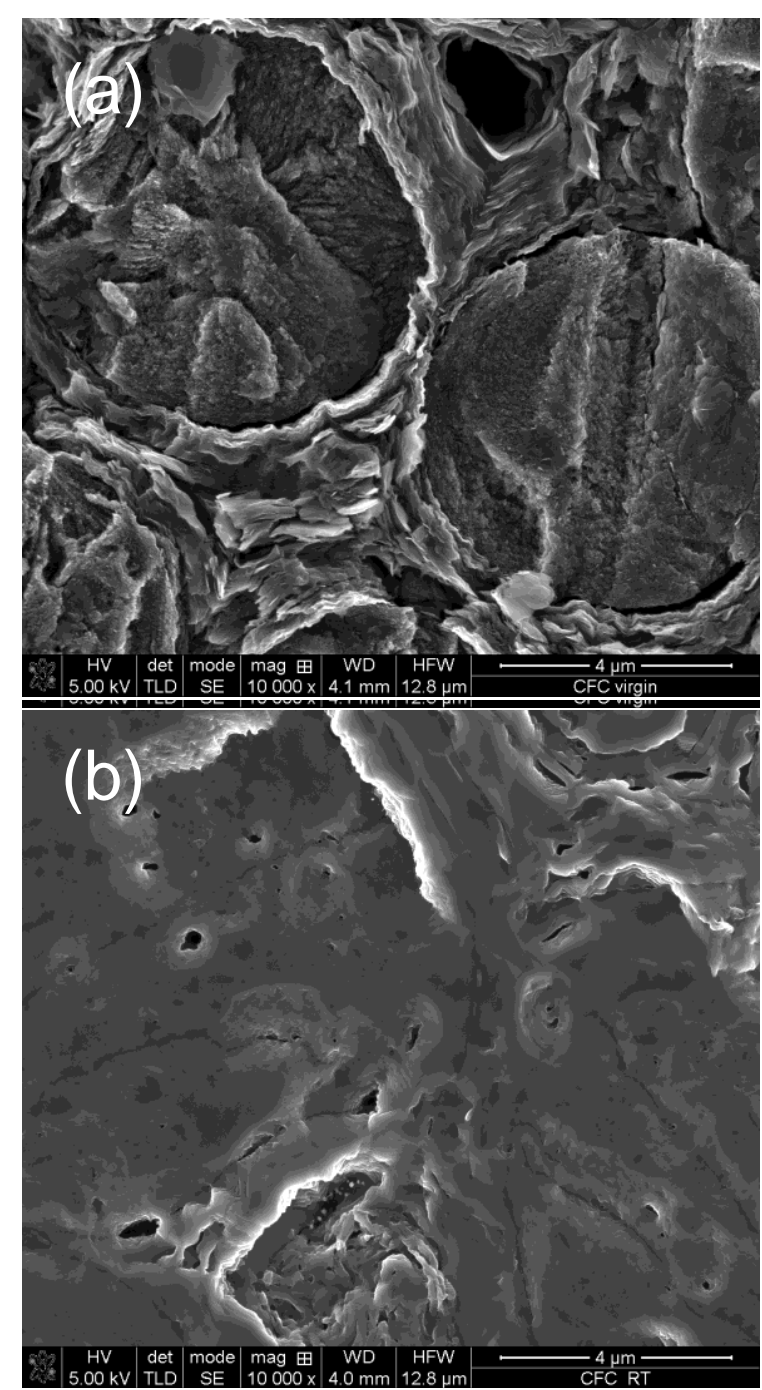

Fig.5. SEM image of the surface of CFC: a) before and b) after plasma irradiation in PlaQ facility at RT, to the fluence of $5 \times 10^{24} \mathrm{D} / \mathrm{m}^{2}$, by $150 \mathrm{eV} / \mathrm{D}$ 